To appear in Physical Review E

\title{
Order Parameter Equations for Front Transitions: Planar and Circular Fronts
}

\author{
A. Hagberg* \\ Center for Nonlinear Studies and T-7, \\ Theoretical Division, Los Alamos National Laboratory, Los Alamos, NM 87545 \\ E. Meron ${ }^{\dagger}$ \\ The Jacob Blaustein Institute for Desert Research and the Physics Department, \\ Ben-Gurion University, Sede Boker Campus 84990, Israel \\ I. Rubinstein and B. Zaltzman \\ The Jacob Blaustein Institute for Desert Research and the Mathematics Department, \\ Ben-Gurion University, Sede Boker Campus 84990, Israel
}

(November 20, 2018)

\begin{abstract}
Near a parity breaking front bifurcation, small perturbations may reverse the propagation direction of fronts. Often this results in nonsteady asymptotic motion such as breathing and domain breakup. Exploiting the time scale differences of an activator-inhibitor model and the proximity to the front bifurcation, we derive equations of motion for planar and circular fronts. The equations involve a translational degree of freedom and an order parameter describing transitions between left and right propagating fronts. Perturbations, such as a space dependent advective field or uniform curvature (axisymmetric spots), couple these two degrees of freedom. In both cases this leads to a transition from stationary to oscillating fronts as the parity breaking bifurcation is approached. For axisymmetric spots, two additional dynamic behaviors are found: rebound and collapse.
\end{abstract}

\section{INTRODUCTION}

Pattern dynamics in reaction-diffusion systems often involve nonsteady front motions. These motions can be driven by curvature [1.2], front interactions [3, 4 , convective instabilities [5] 6 , and external fields [7]. In some cases fronts reverse their direction of propagation, as for example in breathing pulses 10 18, where the reversal is periodic in time, and nucleation of spiral-vortex pairs, where the reversal is local along the extended front line 1923$]$.

Earlier studies demonstrated that front reversals, as described above, become feasible near a nonequilibrium Ising-Bloch (NIB) bifurcation 15.24, that is, a parity breaking bifurcation where a single stationary front loses stability to a pair of new, counterpropagating fronts. The reversal phenomenon can be regarded as a dynamic transition between the left and right propagating fronts that appear beyond the front bifurcation. It is induced by intrinsic perturbations, like curvature and front interactions 19,25], or weak space dependent external fields [20]. Since the left and right propagating fronts differ in internal structure [15] such a transition involves a new degree of freedom in addition to the translation mode: the order parameter associated with the bifurcation 20]. The effect of the perturbations is to couple these two degrees of freedom in a way that allows for front reversal.

Our objective is to derive equations for front motion that capture front reversal. Progress toward that goal has already been made in Ref. [20,26] for a nondiffus- ing inhibitor. Since inhibitor diffusion is essential for spontaneous front reversals induced by curvature, in this work we study the more difficult case of a diffusing inhibitor. This calls for a different approach as described in Sections III and IV. In Section V we study front reversals induced by two types of perturbations of planar fronts, an external advective field and uniform curvature. In this case only planar and circular fronts are studied; the derivation of the more general equations for nonuniformly curved fronts can be found in Ref. [27]. Some of the results presented here have been briefly reported in Ref. [28].

\section{REACTION-DIFFUSION MODEL}

The model we consider is an activator-inhibitor reaction-diffusion system describing a bistable medium. Models of this type have been studied in various physical and chemical contexts [1:2:29 32]. The specific form chosen here is

$$
\begin{aligned}
& u_{t}=\epsilon^{-1}\left(u-u^{3}-v\right)+\delta^{-1} u_{x x}, \\
& v_{t}=u-a_{1} v+v_{x x} .
\end{aligned}
$$

The variables $u$ and $v$ are scalar real fields representing the activator and the inhibitor, respectively, with the subscripts $x$ and $t$ denoting partial derivatives with respect to these variables. For $a_{1}>1$ the system (1) has two stationary uniform states, $\left(u_{ \pm}, v_{ \pm}\right)=$ 


\section{ORDER PARAMETER EQUATIONS FOR FRONT TRANSITIONS}

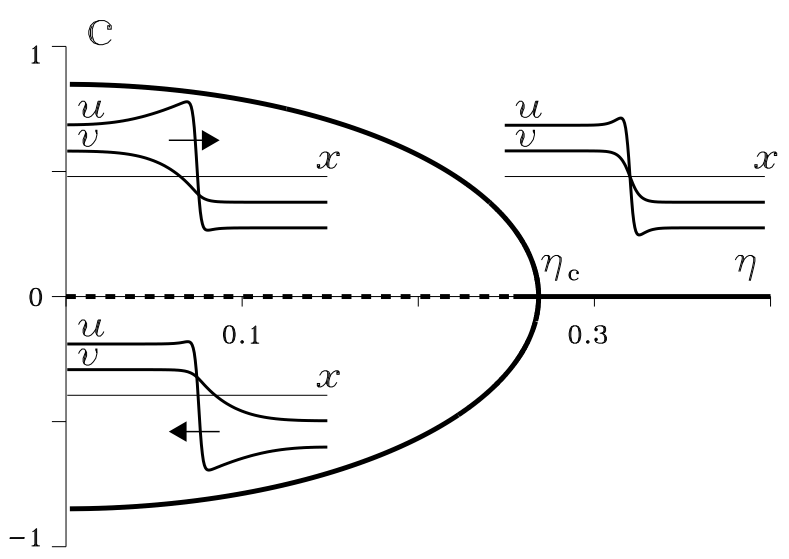

FIG. 1. The NIB (or nonequilibrium Ising-Bloch) bifurcation and internal structure of front solutions. The pitchfork diagram represents the speed of front solutions vs the parameter $\eta$. For $\eta>\eta_{c}$, the Ising front is the single solution and $v_{f}$, the order parameter representing the value of the $v$ field at the front position $u=0$, is zero. Beyond the bifurcation, $\eta<\eta_{c}$, a pair of counterpropagating Bloch fronts appears. The order parameter $v_{f}$ is negative (positive) for rightward (leftward) propagating fronts.

$\left( \pm \sqrt{1-1 / a_{1}}, \pm a_{1}^{-1} \sqrt{1-1 / a_{1}}\right)$. Note that the parity symmetry $(u, v) \rightarrow(-u,-v)$ of Eqs. (11) is reflected in these solutions. Generalizations of Eqs. (1) to nonsymmetric forms in one and two space dimensions will be considered in Section V.

In addition to the spatially uniform solutions there are also front solutions connecting them. In the following we will consider front solutions that connect $\left(u_{+}, v_{+}\right)$at $x=-\infty$ to $\left(u_{-}, v_{-}\right)$at $x=\infty$. The number and type of these front solutions is determined by the two parameters $\epsilon$ and $\delta$. For $\eta>\eta_{c}=3 / 2 \sqrt{2} q^{3}$, where $\eta=\sqrt{\epsilon \delta}$ and $q^{2}=a_{1}+1 / 2$, a single stable stationary (Ising) front solution exists. This solution loses stability in a pitchfork bifurcation, at $\eta=\eta_{c}$, to a pair of counterpropagating (Bloch) front solutions 15,31 33 as shown in Fig. 1. The two Bloch front solutions differ not only in their propagation direction but in their internal structure. This difference can be represented by an order parameter associated with the bifurcation, which for $\mu=\epsilon / \delta \ll 1$ can be taken to be the value $v_{f}$ of the $v$ field at the front position. For simplicity we define the front position to be at $u=0$. With this choice, $v_{f}=0$ for the Ising front (the inset for $\eta>\eta_{c}$ in Fig. 11). Beyond the front bifurcation, $v_{f}$ is nonzero and the sign indicates the direction of front propagation: $v_{f}<0$ for the front propagating to the right and $v_{f}>0$ for the front propagating to the left $\left(\eta<\eta_{c}\right.$ in Fig. 1]).

\section{FORMULATION OF THE FREE BOUNDARY PROBLEM}

In the following we confine ourselves to the region $\epsilon \ll 1, \delta \propto \epsilon^{-1}$ and we choose $\delta$ values such that $\epsilon \delta \sim \mathcal{O}\left(\eta_{c}^{2}\right)$. The small parameter $\epsilon$ allows the use of singular perturbation methods to study front solutions to Eqs. (1). The first step is to transform to a moving coordinate frame, $x \rightarrow r=x-x_{f}(t), t \rightarrow t$ where $x_{f}$ is the position of the front. In this frame Eqs. (11) become

$$
\begin{aligned}
u_{t}-\dot{x}_{f} u_{r} & =\epsilon^{-1}\left(u-u^{3}-v\right)+\delta^{-1} u_{r r}, \\
v_{t}-\dot{x}_{f} v_{r} & =u-a_{1} v+v_{r r},
\end{aligned}
$$

where the dot over $x_{f}$ denotes the derivative with respect to $t$. The front solution, $u(r, t), v(r, t)$, is characterized by a strong variation of the $u$ field over a distance of order $\sqrt{\mu}=\sqrt{\epsilon / \delta}$. Stretching the spatial coordinate, $z=r / \sqrt{\mu}$, to expand this region Eqs. (2) become

$$
\begin{aligned}
\epsilon\left(u_{t}-\dot{z}_{f} u_{z}\right) & =u-u^{3}-v+u_{z z} \\
\mu\left(v_{t}-\dot{z}_{f} v_{z}-u+a_{1} v\right) & =v_{z z}
\end{aligned}
$$

where $z_{f}=x_{f} / \sqrt{\mu}$ and we recall that $\mu \propto \epsilon^{2}$. Expanding in $\epsilon$

$$
\begin{aligned}
& u=u_{0}+\epsilon u_{1}+\epsilon^{2} u_{2}+\ldots, \\
& v=v_{0}+\epsilon v_{1}+\epsilon^{2} v_{2}+\ldots,
\end{aligned}
$$

and inserting into (3) we find at order unity the front solution

$$
u_{0}=-\tanh (z / \sqrt{2}), \quad v_{0}=0 .
$$

Collecting terms of order $\epsilon$ gives

$$
\mathcal{L} u_{1}=v_{1}-\dot{z}_{f} u_{0 z}, \quad \mathcal{L}=\partial_{z}^{2}+1-3 u_{0}^{2},
$$

where $v_{1}$ is a yet undetermined function of time. Since

$$
\mathcal{L} u_{0 z}=0
$$

solvability of (4) requires

$$
\dot{z}_{f}=-\frac{3}{\sqrt{2}} v_{1}(t) .
$$

The narrow front region becomes infinitely thin in the limit $\epsilon \rightarrow 0$. Therefore, $v(t)$ may be associated with the value of $v(r, t)$ at the front position, that is $v(0, t)$. With this notation the leading order relation is

$$
\dot{x}_{f}=-\frac{3}{\eta \sqrt{2}} v(0, t) .
$$

Away from $x_{f}, u-u^{3}-v \sim \mathcal{O}(\epsilon)$, and $u$ varies on the same time and length scales as $v$. Going back to Eqs. (2) we find at order unity 


\section{A. HAGBERG, E. MERON, I. RUBINSTEIN, AND B. ZALTZMAN}

$$
\begin{array}{ll}
v_{t}-\dot{x}_{f} v_{r}=u_{+}(v)-a_{1} v+v_{r r}, & r \leq 0, \\
v_{t}-\dot{x}_{f} v_{r}=u_{-}(v)-a_{1} v+v_{r r}, & r \geq 0,
\end{array}
$$

where $u_{ \pm}(v)$ are the outer solution branches of the cubic equation $u-u^{3}-v=0$. For $a_{1}$ sufficiently large we may linearize the branches $u_{ \pm}(v)$ around $v=0$

$$
u_{ \pm}(v) \approx \pm 1-v / 2 .
$$

Substituting the linearization (7) and the relation from the inner problem (5) into (6) produces the free boundary problem

$$
\left.\begin{array}{rl}
v_{t}+q^{2} v-v_{r r} & =1-\frac{3}{\eta \sqrt{2}} v(0, t) v_{r} \\
v(-\infty, t) & =v_{+} \approx q^{-2}
\end{array}\right\} \quad r \leq 0
$$

where the square brackets in (9) denote jumps across the free boundary. The solution to (8) leads to a dynamic equation for $v_{f}(t)=v(0, t)$, the value of the inhibitor at the front position $x_{f}(t)$, which will complement Eq. (5).

\section{SOLUTION OF THE FREE BOUNDARY PROBLEM}

Near the front bifurcation ( $\eta$ close to $\eta_{c}$ ), the front speed, $c$, is small and propagating front solutions can be expanded as power series in $c$ around the stationary front solution. The stationary front solution satisfies the boundary value problem

$$
\left.\begin{array}{rl}
v_{r r}-q^{2} v+1 & =0 \\
v(-\infty) & =q^{-2} \\
v(0) & =0
\end{array}\right\} \quad r \leq 0,
$$

with $\left[v_{r}\right]_{r=0}=0$. The solution to (10) is

$$
\begin{array}{ll}
v^{(0)}(r)=q^{-2}\left(1-e^{q r}\right), & r \leq 0, \\
v^{(0)}(r)=q^{-2}\left(e^{-q r}-1\right), & r \geq 0 .
\end{array}
$$

Note that $v_{r}^{(0)}=-q^{-1} \exp (-q|r|)$ is not differentiable at $r=0$.

In terms of the deviation from the stationary solution, $\bar{v}=v-v^{(0)}$, Eqs. (8) are

$$
\begin{aligned}
\bar{v}_{t}+q^{2} \bar{v}-\bar{v}_{r r} & =-\frac{3}{\eta \sqrt{2}} v(0, t)\left(\bar{v}_{r}+v_{r}^{(0)}\right), \\
\bar{v}( \pm \infty) & =0
\end{aligned}
$$

We seek propagating solutions of (12) that involve two time scales, the original time $t$ and a slow time $T=c^{2} t$. The slow time dependence is a result of the slow evolution of $v_{f}$ (the value of the inhibitor at the front position) near the front bifurcation. It is easy to show that to linear order $\dot{v}_{f} \propto\left(\eta_{c}-\eta\right) v_{f}$ and for a pitchfork bifurcation $\eta_{c}-\eta \propto c^{2}$, hence the $c^{2}$ scale. Expanding $\bar{v}(r, t, T)$ in powers of $c$ and $\eta$ in powers of $c^{2}$ (expecting a pitchfork bifurcation),

$$
\bar{v}(r, t, T)=\sum_{n=1}^{\infty} c^{n} v^{(n)}(r, t, T),
$$

$$
\eta=\eta_{c}-c^{2} \eta_{1}+c^{4} \eta_{2}+\ldots,
$$

and inserting in (12) gives the sequence of equations

$$
v_{t}^{(n)}+q^{2} v^{(n)}-v_{r r}^{(n)}=-\rho^{(n)}, \quad n=1,2,3,
$$

where

$$
\begin{aligned}
\rho^{(1)}= & \frac{3}{\sqrt{2} \eta_{c}} v_{\mid r=0}^{(1)} v_{r}^{(0)}, \\
\rho^{(2)}= & \frac{3}{\sqrt{2} \eta_{c}}\left[v_{\mid r=0}^{(1)} v_{r}^{(1)}+v_{\mid r=0}^{(2)} v_{r}^{(0)}\right] \\
\rho^{(3)}= & v_{T}^{(1)}+\frac{3 \eta_{1}}{\sqrt{2} \eta_{c}^{2}} v_{\mid r=0}^{(1)} v_{r}^{(0)} \\
& +\frac{3}{\sqrt{2} \eta_{c}}\left[v_{\mid r=0}^{(1)} v_{r}^{(2)}+v_{\mid r=0}^{(2)} v_{r}^{(1)}+v_{\mid r=0}^{(3)} v_{r}^{(0)}\right] .
\end{aligned}
$$

Equation (15) can be solved using an appropriate Green's function and assuming that the front motion is independent of the fast time scale $t$ as $t \rightarrow \infty$ [28]. A simplified derivation of the solution follows from the gradient nature of (15) when the source term $\rho^{(n)}(r, T)$ becomes independent of $t$. For then $v_{t}^{(n)} \rightarrow 0$ as $t \rightarrow \infty$ for any $r$ and we can look for stationary ( $t$ independent) solutions.

Consider first $v^{(1)}$. Inserting (16a) in $v_{r r}^{(1)}-q^{2} v^{(1)}=$ $\rho^{(1)}$ and solving for $v^{(1)}$ we obtain

$$
v^{(1)}(r, T)=\frac{3}{2 \sqrt{2} \eta_{c} q^{3}} v^{(1)}(0, T) F(r),
$$

where

$$
\begin{array}{ll}
F(r)=(1-q r) e^{q r}, & r \leq 0, \\
F(r)=(1+q r) e^{-q r}, & r \geq 0 .
\end{array}
$$




\section{ORDER PARAMETER EQUATIONS FOR FRONT TRANSITIONS}

Setting $r=0$ we find

$$
\eta_{c}=\frac{3}{2 \sqrt{2} q^{3}} .
$$

The critical value $\eta_{c}=\eta(c=0)$ determines the bifurcation point where the propagating Bloch front solutions coincide with the stationary Ising front. The expression (18) is the same as the one derived earlier using a different method 15,25 .

Using (17) to solve $v_{r r}^{(2)}-q^{2} v^{(2)}=\rho^{(2)}$ we find

$$
v^{(2)}(r, T)=\left(v^{(2)}(0, T)+\frac{1}{2} v^{(1)}(0, T)^{2} q^{3} r\right) F(r),
$$

and using both $(17)$ and (19) in $v_{r r}^{(3)}-q^{2} v^{(3)}=\rho^{(3)}$ we obtain

$$
\begin{aligned}
& \left.\begin{array}{rl}
v^{(3)}(r, T)= & v^{(3)}(0, T) e^{q r}+A_{+} r e^{q r} \\
& -B_{+} r^{2} e^{q r}-C_{+} r^{3} e^{q r},
\end{array}\right\} r \leq 0, \\
& \left.\begin{array}{rl}
v^{(3)}(r, T)= & v^{(3)}(0, T) e^{-q r}+A_{-} r e^{-q r} \\
& -B_{-} r^{2} e^{-q r}-C_{-} r^{3} e^{-q r},
\end{array}\right\} r \geq 0,
\end{aligned}
$$

where

$$
\begin{aligned}
A_{ \pm}= & q^{3} v^{(1)}(0, T) v^{(2)}(0, T) \\
& \pm\left[\frac{3}{4 q} v_{T}^{(1)}(0, T)+\frac{1}{2} q^{5} v^{(1)}(0, T)^{3}\right. \\
& \left.-\frac{q \eta_{1}}{\eta_{c}} v^{(1)}(0, T)-q v^{(3)}(0, T)\right], \\
B_{ \pm}= & \frac{1}{4} v_{T}^{(1)}(0, T) \pm q^{4} v^{(1)}(0, T) v^{(2)}(0, T), \\
C_{ \pm}= & \pm \frac{1}{6} q^{7} v^{(1)}(0, T)^{3} .
\end{aligned}
$$

Application of the (no) jump condition $\left[v_{r}^{(3)}\right]_{r=0}=0$ leads to

$$
\eta_{c}^{2} v_{T}^{(1)}(0, T)=\frac{\sqrt{2} \eta_{1}}{q} v^{(1)}(0, T)-\frac{3}{4} v^{(1)}(0, T)^{3} .
$$

Equation (21) still contains an unspecified parameter, $\eta_{1}$. To identify $\eta_{1}$ recall that $c$ is the speed of a front propagating at constant velocity. From (5)

$$
\left|\dot{x}_{f}\right|=\frac{3}{\sqrt{2} \eta_{c}} c\left|v^{(1)}(0, T)\right|+\mathcal{O}\left(c^{2}\right) .
$$

Identifying $\left|\dot{x}_{f}\right|$ with $c$ gives $v^{(1)^{2}}=2 \eta_{c}^{2} / 9$ for a front propagating at constant speed. This value of $v^{(1)^{2}}$ should coincide with the nontrivial stationary solution of (21), $v^{(1)^{2}}=4 \sqrt{2} \eta_{1} / 3 q$. Comparing the two expressions gives

$$
\eta_{1}=\frac{q \eta_{c}^{2}}{6 \sqrt{2}}
$$

Equations (22) and (14) provide the leading order form of the front bifurcation diagram

$$
c^{2}=\frac{6 \sqrt{2}}{q \eta_{c}^{2}}\left(\eta_{c}-\eta\right)
$$

which coincides for small $c$ with the earlier result $[6,9]$, $c^{2}=4 q^{2}\left(\eta_{c}^{2}-\eta^{2}\right) / \eta^{2}$.

Multiplying (21) by $c$ and using the expansions (13) and (14) gives the equation of motion for propagating fronts

$$
\begin{aligned}
& \dot{v}_{f}=\frac{\sqrt{2}}{q \eta_{c}^{2}}\left(\eta_{c}-\eta\right) v_{f}-\frac{3}{4 \eta_{c}^{2}} v_{f}^{3}, \\
& \dot{x}_{f}=-\frac{3}{\eta \sqrt{2}} v_{f},
\end{aligned}
$$

where the slow time derivative of $v_{f}$ is expressed in terms of a fast time derivative $\left(\dot{v}_{f}=c^{2} v_{f}\right)$.

According to Eqs. (23) the dynamics of a propagating front involve two degrees of freedom: a translational degree of freedom, $x_{f}$, determining the front position, and an order parameter, $v_{f}$, determining the direction of propagation. The latter has not been appreciated enough since most works to date [1.2] have focused on conditions far from the front bifurcation. In that case the two stationary states, $v_{f}= \pm\left[4 \sqrt{2}\left(\eta_{c}-\eta\right) / 3 q\right]^{-1 / 2}$, representing fronts propagating in opposite directions, are highly stable. Close to the front bifurcation, however, the eigenvalue associated with these states, $\lambda=-2 \sqrt{2}\left(\eta_{c}-\eta\right) / q$, approaches zero and small disturbances can drive the system from one state to another, thereby inducing front reversals.

\section{FRONT REVERSAL: OSCILLATIONS AND REBOUND}

Equations (23) describe the motion of a freely propagating front in a uniform medium. In this Section we show how two different perturbations affect front propagation. The first is the addition of a space dependent advective field to the $v$ equation in the original system (11). This type of differential advection appears for example in chemical reactions involving ionic species that are subjected to electric fields [8,34]. The second is the intrinsic perturbation of uniform curvature variations on the propagation of two dimensional fronts. Both perturbations lead to a coupling of the two degrees of freedom in the order parameter Eqs. (23) and allow for the nonsteady asymptotic motion of fronts. 


\section{A. HAGBERG, E. MERON, I. RUBINSTEIN, AND B. ZALTZMAN}

\section{A. Space dependent advective field}

To study the effect of an external advective field $J$ we add the term $J v_{x}$ to the $v$ equation in (1),

$$
\begin{aligned}
& u_{t}=\epsilon^{-1}\left(u-u^{3}-v\right)+\delta^{-1} u_{x x}, \\
& v_{t}=u-a_{1} v-a_{0}+J v_{x}+v_{x x} .
\end{aligned}
$$

The small parameter $a_{0}$ is also introduced to break the parity symmetry of (11). For simplicity we consider a linear spatial profile, $J=-\alpha x, 0<\alpha \ll 1$. Proceeding as before, the inner region analysis remains unchanged and culminates in $(23 \mathrm{~b})$. The outer analysis leads to the additional terms, $-\alpha\left(r+x_{f}\right) v_{r}-a_{0}$, on the right hand side of both partial differential equations in (8). Assuming $\alpha=\alpha_{0} c^{3}$ and $a_{0}=a_{00} c^{3}$, where $\alpha_{0}$ and $a_{00}$ are of order unity, Eqs. (16a) and (16b) remain unchanged, but (16c) acquires on the right hand side two additional terms: $\alpha_{0}\left(r+x_{f}\right) v_{r}^{(0)}+a_{00}$. As a result the order parameter equations (23) are changed to

$$
\begin{aligned}
& \dot{v}_{f}=\frac{\sqrt{2}}{q \eta_{c}^{2}}\left(\eta_{c}-\eta\right) v_{f}-\frac{3}{4 \eta_{c}^{2}} v_{f}^{3}+\frac{2}{3 q} \alpha x_{f}-\frac{4}{3} a_{0}, \\
& \dot{x}_{f}=-\frac{3}{\eta \sqrt{2}} v_{f} .
\end{aligned}
$$

Notice that the introduction of a space dependent advective field couples the two degrees of freedom, $v_{f}$ and $x_{f}$. This coupling affects the front behavior in two significant ways: for $\eta>\eta_{c}$ (and $a_{0} \neq 0$ ) it stabilizes a propagating front at a fixed position, $x_{f}=2 q a_{0} / \alpha$, and for $\eta<\eta_{c}$ it induces oscillations between the counterpropagating fronts. The frequency of oscillations close to the Hopf bifurcation at $\eta=\eta_{c}$ is

$$
\omega=\frac{2}{\sqrt{3}} q \sqrt{\alpha} .
$$

We tested the validity of Eqs. (25) by numerically integrating the original system (11) and comparing the oscillating front solutions with those of (25). The agreement as Fig. 2 shows is very good. In Fig. 3 we plotted the frequency of front oscillations vs the field gradient according to (26) and as obtained from (11). Again, the agreement is excellent, and remains good even for $c$ of order unity. Note that in the inner analysis we neglected contributions of $\mathcal{O}\left(\epsilon^{2}\right)$ to $v$ at the front position, while in the outer analysis we kept terms to $\mathcal{O}\left(c^{3}\right)$. A quantitative comparison as described above therefore requires that $c$ is much larger than $\epsilon^{2 / 3}$.

\section{B. Uniform curvature}

In two space dimensions the reaction-diffusion system (11) becomes

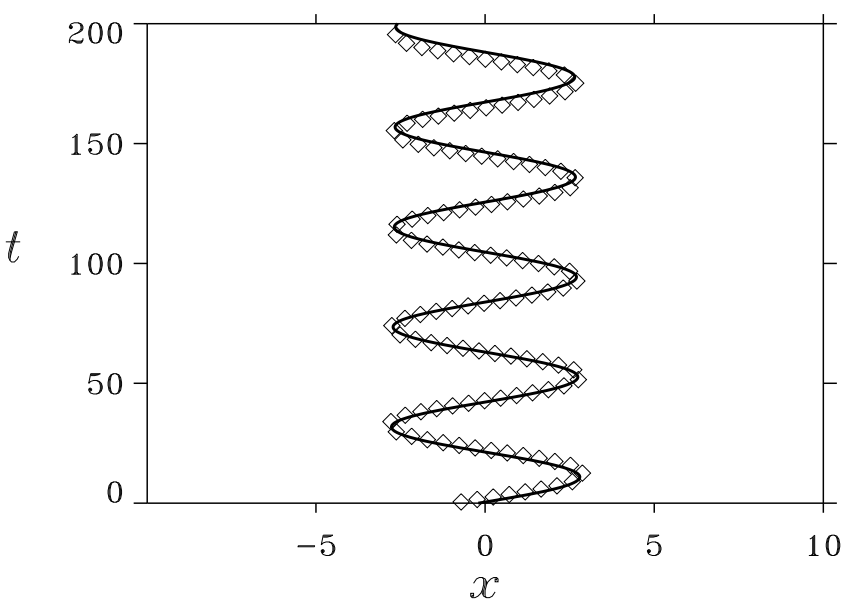

FIG. 2. Front position, $x_{f}$, vs time for an oscillating front. The solid line represents the solution to the order parameter equations (25) and the diamonds are from the numerical solution of the original partial differential equations (11). Parameters: $a_{1}=3.0, \epsilon=0.01, \delta=2.77, a_{0}=0, \alpha=0.005$.

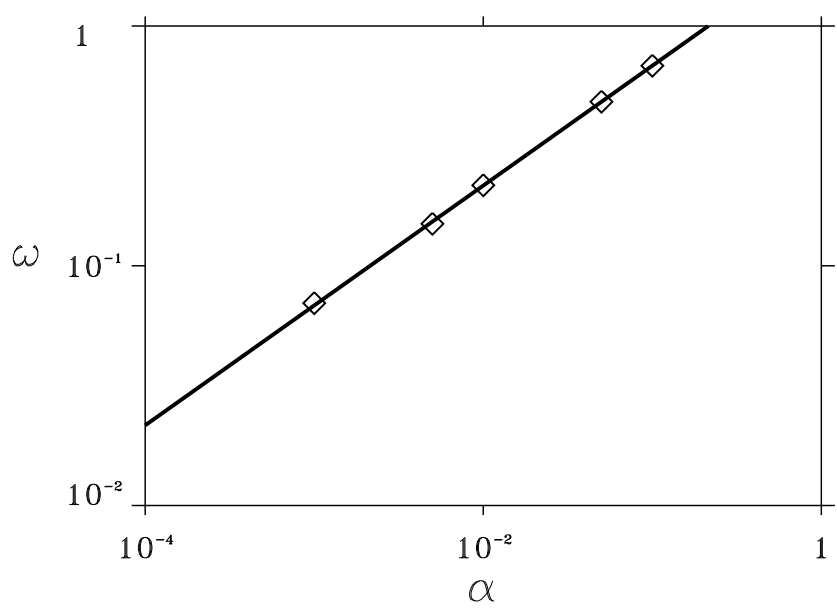

FIG. 3. A log-log plot of the oscillation frequency, $\omega$, vs the external field gradient, $\alpha$. The solid line is the relation of Eq. (26) and the diamonds represent numerical solutions of Eqs. (11). Parameters: $a_{1}=3.0, \epsilon=0.01, \delta=2.77, a_{0}=0$. 


\section{ORDER PARAMETER EQUATIONS FOR FRONT TRANSITIONS}

$$
\begin{aligned}
& u_{t}=\epsilon^{-1}\left(u-u^{3}-v\right)+\delta^{-1} \nabla^{2} u \\
& v_{t}=u-a_{1} v-a_{0}+\nabla^{2} v
\end{aligned}
$$

where the small parameter $a_{0}$ has been added again to break the parity symmetry of (1). In addition to planar front solutions there are now new types of solutions including fronts with uniform curvature (circular fronts or spots). These spots may be stationary or, for parameters near a NIB bifurcation, may collapse, expand indefinitely, or oscillate periodically in time.

To derive equations for the motion of circular fronts, the first step is to transform into polar coordinates, $r=$ $\rho-\rho_{f}(t)$, that move with the front. In this frame and assuming the radius of curvature $\rho_{f}$ is much larger than the front width, Eqs. (27) are

$$
\begin{aligned}
u_{t}-\left(\dot{\rho}_{f}+\delta^{-1} \kappa\right) u_{r} & =\epsilon^{-1}\left(u-u^{3}-v\right)+\delta^{-1} u_{r r}, \\
v_{t}-\left(\dot{\rho}_{f}+\kappa\right) v_{r} & =u-a_{1} v-a_{0}+v_{r r}
\end{aligned}
$$

where $\kappa=\rho_{f}^{-1}$ is the front curvature. As before we assume $\epsilon \ll 1$ and $\delta \propto \epsilon^{-1}$ and use singular perturbation theory.

Analysis of the inner, or front, region yields a relation analogous to (23b)

$$
\dot{\rho}_{f}+\delta^{-1} \kappa=-\frac{3}{\eta \sqrt{2}} v_{f}
$$

In the outer region, instead of $(8)$ we must solve

$$
v_{t}+q^{2} v-v_{r r}= \pm 1-\frac{3}{\eta \sqrt{2}} v(0, t) v_{r}+P
$$

where $P=\left(1-\delta^{-1}\right) v_{r} / \rho_{f}-a_{0}$. Assuming that $P$ is a small perturbation (of order $|c|^{3}$ ) and proceeding as in Section [V] we obtain the order parameter equation

$$
\dot{v}_{f}=\frac{\sqrt{2}}{q \eta_{c}^{2}}\left(\eta_{c}-\eta\right) v_{f}-\frac{3}{4 \eta_{c}^{2}} v_{f}^{3}-\frac{2}{3 q} \frac{\left(1-\delta^{-1}\right)}{\rho_{f}}-\frac{4}{3} a_{0} .
$$

Writing Eqs. (28) and (29) terms of the curvature $\kappa=$ $\rho_{f}^{-1}$ gives the equations

$$
\begin{aligned}
\dot{v}_{f} & =\frac{\sqrt{2}}{q \eta_{c}^{2}}\left(\eta_{c}-\eta\right) v_{f}-\frac{3}{4 \eta_{c}^{2}} v_{f}^{3}-\frac{2}{3 q}\left(1-\delta^{-1}\right) \kappa-\frac{4}{3} a_{0} \\
\dot{\kappa} & =\frac{3}{\eta \sqrt{2}} v_{f} \kappa^{2}+\delta^{-1} \kappa^{3}
\end{aligned}
$$

that describe the dynamics of large circular spots. The introduction of curvature couples the two equations. Equations (23) for planar fronts are decoupled and only describe the relaxation to steadily propagating fronts. The equations for circular fronts additionally allow front reversals and nonsteady asymptotic motion like oscillations.

Consider first the fixed point solutions obtained by the intersections of the linear nullclines $\kappa=0$ and $\kappa=$
$-(3 \delta / \eta \sqrt{2}) v_{f}$ of $(30 \mathrm{~b})$ with the cubic nullcline of (30a). The solutions corresponding to $\kappa=0$ describe planar fronts propagating at constant velocities. Solutions with positive and negative $v_{f}$ values pertain to down states invading up states and up states invading down states, respectively. The number of $\kappa=0$ solutions varies with $\eta$. Below the front bifurcation, $\left[\eta>\eta_{c}\left(a_{0}\right)\right]$, there is a single intersection point representing an Ising front as shown by the thin lines in Figs. A a and $4 \mathrm{~b}$. Beyond the front bifurcation, $\left[\eta<\eta_{c}\left(a_{0}\right)\right]$, two more intersection points appear corresponding a stable and unstable pair of planar front solutions (Fig. 4c). The fixed point solutions for $\kappa \neq 0$ represent a circular fronts. For $a_{0}<0$ they describe spots of up an state domain and for $a_{0}>0$ spots of a down state domain. For $\delta>1$, depending on the choice of $\epsilon$, these fixed points may or may not be stable. For $\delta<1$, all the $\kappa \neq 0$ fixed points are unstable.

Figure 4 shows three different possibilities for the dynamics of circular fronts. The thick trajectories represent dynamics computed by numerical solution of the coupled equations (30). The initial conditions correspond to a large shrinking up state spot. Far into the Ising regime (Fig. 4a) the initial spot converges to a stationary spot. Moving closer to the front bifurcation and past a critical $\eta$ value, $\eta_{H}>\eta_{c}\left(a_{0}\right)$, a Hopf bifurcation to a breathing spot occurs (Fig. $4 \mathrm{~b})$. Crossing the front bifurcation, $\eta<\eta_{c}\left(a_{0}\right)$, the spot rebounds, i.e. the shrinking spot reaches a minimal size and expands again indefinitely (Fig. A $\mathrm{c}$ ). For larger $\left|a_{0}\right|$, there is another possibility for the dynamics of spots. In this case, shown in Fig. 5, the amplitude of oscillations grows in time until the spot eventually collapses as the curvature diverges to infinity.

All the three behaviors discussed above have been observed in direct numerical solutions of (27). The quantitative accuracy of the order parameter equations was tested by computing numerical solutions to the circularly symmetric version of Eqs. 27)

$$
\begin{aligned}
& u_{t}=\epsilon^{-1}\left(u-u^{3}-v\right)+\frac{\delta^{-1}}{r} u_{r}+\delta^{-1} u_{r r}, \\
& v_{t}=u-a_{1} v-a_{0}+\frac{1}{r} v_{r}+v_{r r} .
\end{aligned}
$$

and comparing them to solutions to Eqs. (30) for spot dynamics. Spot solutions of (31) produce the same qualitative behavior as the pair of coupled equations for the spot dynamics. When the parameters are chosen to satisfy the assumptions made in the derivation of (30), there is also quantitative agreement between the two solutions. Figure 6 shows the curvature of an oscillating spot as a function of time computed using both Eqs. (30) and (31). The two solutions agree within an accuracy of approximately $1 \%$ for the amplitude and $2 \%$ for the phase.

In addition to the oscillatory instability spot solutions may also be unstable to transverse perturbations 35 37. Numerical solutions of the fully twodimensional model (27) show that for the parameters of Fig. 6 spots are unstable and form nonuniformly curved 


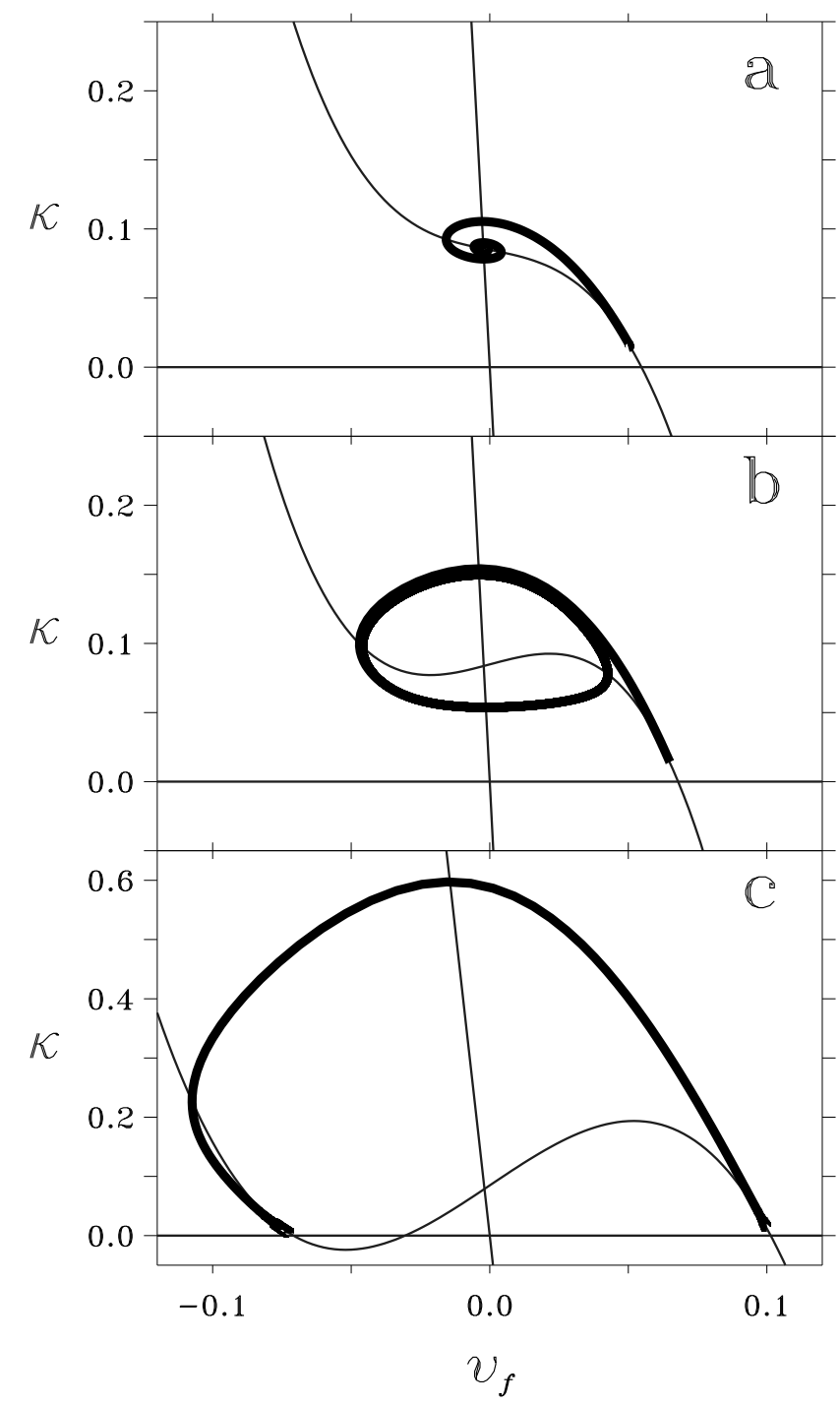

FIG. 4. Three types of solutions to the order parameter equations (30) starting with initial conditions representing a large shrinking spot. The thin lines are the isoclines and the thick lines are numerically computed trajectories. (a) Convergence to a stationary spot $(\epsilon=.0063)$. (b) An oscillating spot $(\epsilon=.006)$. (c) Spot rebound and expansion of the spot to infinite size $(\epsilon=.0052)$. Parameters: $a_{1}=4.0, a_{0}=-0.01$, and $\delta=2.0$.

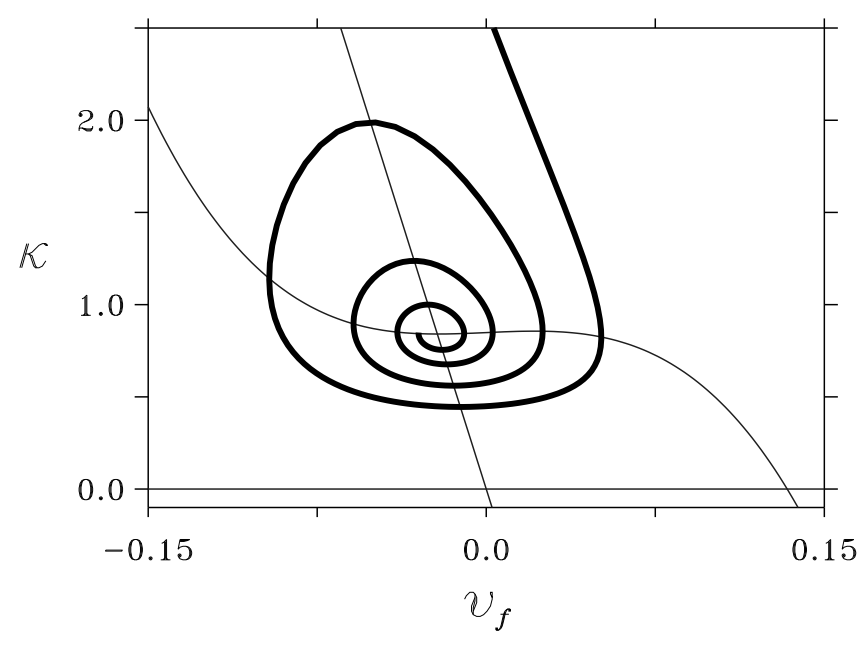

FIG. 5. A trajectory of the order parameter equations (30) for a spot that oscillates with growing amplitude until collapse (the curvature $\kappa$ diverges to infinity). Parameters: $a_{1}=4.0$, $a_{0}=-0.1, \epsilon=0.006$, and $\delta=2.0$.

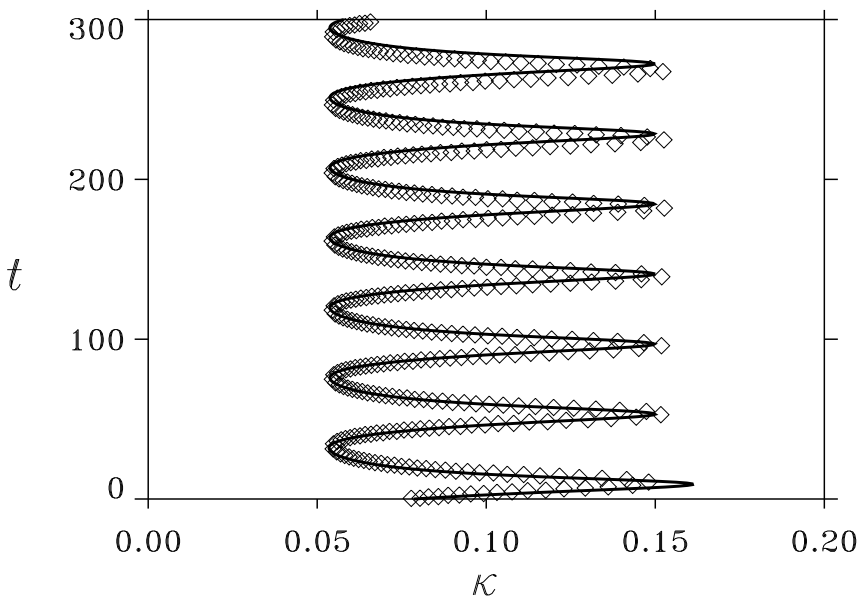

FIG. 6. An oscillating circular spot solution. The solid line is the solution of the order parameter equations (30), and the diamonds represent the spot curvature vs time from the numerical solution to the circularly symmetric equations (31). The equation parameters are $\epsilon=0.006, \delta=2.0, a_{1}=4.0, a_{0}=-0.01$. 


\section{ORDER PARAMETER EQUATIONS FOR FRONT TRANSITIONS}

fronts leading to a labyrinthine pattern. Since the order parameter equations derived here apply only for the case when the spots do not break perfect circular symmetry, for this choice of parameters they only capture the dynamics of the circular spot during the initial evolution. Order parameter equations for the dynamics of nonuniformly curved fronts are presented in Ref. 27].

\section{CONCLUSION}

We derived the equations that govern the dynamics of planar fronts in bistable systems near a parity breaking front bifurcation (the NIB bifurcation). In this case the context is an activator-inhibitor model, but the normal form

$$
\begin{aligned}
\dot{X} & =C, \\
\dot{C} & =\left(\alpha_{c}-\alpha\right) C-\beta C^{3},
\end{aligned}
$$

is general. Here, $X$ is the front position, $C$ is the front velocity, and $\alpha_{c}$ is a critical parameter value for which a NIB bifurcation occurs. Similar equations should apply, for example, to liquid crystals subjected to rotating magnetic fields [38 40] and have also been proposed in the context of parity breaking traveling-wave bifurcations 41.

Uniform front curvature, or space dependent external fields, couple the two degrees of freedom, $X$ and $C$, and allow nonsteady asymptotic behavior. The coupled sets of equations (25) and (30) exhibit Hopf bifurcations from stationary to oscillating fronts (breathing spots). Equations (30) exhibit two additional behaviors pertaining to rebounding and collapsing spots in the full equations.

Curvature effects on front dynamics near a NIB bifurcation were also studied in Refs. 20,25] using a "quasistatic" approximation. This approximation, where the front velocity is assumed to adiabatically follow slow curvature variations [1.24, yields an algebraic relation between the front velocity $C$ and its curvature $\kappa$. As the bifurcation is approached the $C-\kappa$ relation becomes multivalued, or hysteretic. The multivalued relations correctly predict spontaneous front transitions induced by curvature [20,25], but cannot describe dynamics during front transitions. Differential order parameter equations, like (30), give a more accurate characterization of the dynamics. These differential equations reduce to an algebraic $C-\kappa$ relation when the time scale of front transitions becomes much shorter than the time scale of curvature changes. Such a condition is realized, for example, with very large spots away from the front bifurcation. Then the right hand side of (30a) can be set to zero, an expression which together with (28) gives an algebraic $C-\kappa$ relation where $C=\dot{\rho}_{f}$.

The phenomena of breathing, oscillating, and collapsing spots appear to be quite general and can be induced by other perturbations that couple $X$ and $C$. Ref. [42], which studies the effect of boundaries on spot dynamics, reports on the observation of stationary, breathing, and rebounding spots. Interaction between fronts may similarly lead to stationary, oscillating, and collapsing domains [10 18]. Recent experiments on the FerrocyanideIodate-Sulfite reaction show small oscillating chemical spots away from the reactor boundary that are most likely due to front interactions and/or curvature [43].

\section{ACKNOWLEDGMENTS}

We thank the anonymous referee for suggesting a shorter method for the solution of Eqs. (15). This study was supported in part by the Israel Ministry of Science and the Arts.

[1] J. J. Tyson and J. P. Keener, Physica D 32, 327 (1988).

[2] E. Meron, Physics Reports 218, 1 (1992).

[3] C. Elphick, E. Meron, and E. A. Spiegel, Phys. Rev. Lett. 61, 496 (1988).

[4] C. Elphick, E. Meron, and E. A. Spiegel, SIAM J. Appl. Math 50, 490 (1990).

[5] K. I. Agladze, V. I. Krinsky, and A. M. Pertsov, Nature 308, 834 (1984).

[6] S. C. Müller, T. Plesser, and B. Hess, in Physiochemical Hydrodynamics: Interfacial Phenomena, edited by M. G. Velarde (Plenum Press, New York, 1988).

[7] P. Ortoleva, Physica D 26, 67 (1987).

[8] O. Steinbock, J. Schütze, and S. C. Müller, Phys. Rev. Lett. 68, 248 (1992).

[9] A. F. Munster, P. Hasal, and M. Marek, Phys. Rev. E 50, 546 (1994).

[10] S. Koga and Y. Kuramoto, Prog. Theor. Phys. 63, 106 (1980).

[11] B. S. Kerner and V. V. Osipov, Sov. Phys. JETP 56, 1275 (1982).

[12] Y. Nishiura and M. Mimura, SIAM J. Appl. Math. 49, 481 (1989).

[13] T. Ohta, A. Ito, and A. Tetsuka, Phys. Rev. A 42, 3225 (1990).

[14] Y. A. Rzanov, H. Richardson, A. A. Hagberg, and J. V. Moloney, Phys. Rev. A 47, 1480 (1993).

[15] A. Hagberg and E. Meron, Nonlinearity 7, 805 (1994).

[16] M. Suzuki, T. Ohta, M. Mimura, and H. Sakaguchi, Phys. Rev. E 52, 3645 (1995).

[17] C. B. Muratov and V. V. Osipov, Phys. Rev. E 53, 3101 (1996).

[18] R. Woesler, P. Schütz, M. Bode, M. Or-Guil, and H.-G. Purwins, Physica D 91, 376 (1996).

[19] A. Hagberg and E. Meron, Phys. Rev. Lett. 72, 2494 (1994).

[20] C. Elphick, A. Hagberg, and E. Meron, Phys. Rev. E 51, 3052 (1995). 
[21] K. J. Lee, W. D. McCormick, Q. Ouyang, and H. L. Swinney, Science 261, 192 (1993).

[22] K. J. Lee, W. D. McCormick, J. E. Pearson, and H. L. Swinney, Nature 369, 215 (1994).

[23] K. J. Lee and H. L. Swinney, Phys. Rev. E 51, 1899 (1995).

[24] P. Coullet, J. Lega, B. Houchmanzadeh, and J. Lajzerowicz, Phys. Rev. Lett. 65, 1352 (1990).

[25] A. Hagberg and E. Meron, Chaos 4, 477 (1994).

[26] M. Bode, "Front bifurcations in reaction-diffusion systems with inhomogeneous parameters" Submitted to Physica D (1996) (unpublished).

[27] A. Hagberg and E. Meron, "The dynamics of curved fronts: Beyond geometry", to appear in Physical Review Letters (unpublished).

[28] A. Hagberg, E. Meron, I. Rubinstein, and B. Zaltzman, Phys. Rev. Lett. 76, 427 (1996).

[29] M. Bär, S. Nettesheim, H. H. Rotermund, M. Eiswirth, and G.Ertl, Phys Rev. Lett. 74, 1246 (1995).

[30] G. Haas, M. Bär, I. G. Kevrekidis, P. B. Rasmussen, H.-H. Rotermund, and G.Ertl, Phys Rev. Lett. 75, 3560 (1995).

[31] M. Bode, A. Reuter, R. Schmeling, and H.-G. Purwins, Phys Lett. A 185, 70 (1994).

[32] P. Schütz, M. Bode, and H.-G. Purwins, Physica 82D, 382 (1995).

[33] H. Ikeda, M. Mimura, and Y. Nishiura, Nonl. Anal. TMA 13, 507 (1989).

[34] J. J. Taboada, A. P. Muñuzuri, V. Pérez-Muñuzuri, M. Gómez-Gesteira, and V. Pérez-Villar, Chaos 4, 519 (1994).

[35] T. Ohta, M. Mimura, and R. Kobayashi, Physica D 34, 115 (1989).

[36] D. M. Petrich and R. E. Goldstein, Phys. Rev. Lett. 72, 1120 (1994).

[37] R. E. Goldstein, D. J. Muraki, and D. M. Petrich, Phys. Rev. E 53, 3933 (1996).

[38] S. Nasuno, N. Yoshimo, and S. Kai, Phys. Rev. E 51, 1598 (1995).

[39] T. Frisch, S. Rica, P. Coullet, and J. M. Gilli, Phys. Rev. Lett. 72, 1471 (1994).

[40] K. B. Migler and R. B. Meyer, Physica D 71, 412 (1994).

[41] E. Knobloch, J. Hettel, and G. Danglemayr, Phys. Rev. Lett. 74, 4839 (1995).

[42] D. Haim, G. Li, Q. Ouyang, W. D. McCormick, H. L. Swinney, A. Hagberg, and E. Meron, Phys. Rev. Lett. 77, 190 (1996).

[43] G. Li, Q. Ouyang, and H. L. Swinney, J. Chem. Phys 105, 10830 (1996). 